\title{
La depreciación de los derechos humanos y la regulación de la reproducción humana asistida en México
}

\author{
Rosa Verónica Esparza-Pérez \\ Doctoranda, Universidad Nacional de Educación a Distancia, Madrid, España
}

\begin{abstract}
Resumen
La infertilidad representa un problema en el contexto de la salud reproductiva. Para tratarla se desarrollaron las técnicas de reproducción humana asistida; a pesar de los avances médicos, su práctica está sujeta a disfunciones médicas, legales y éticas. En México, desde finales de la década de 1980 se crearon clínicas de reproducción asistida en hospitales del sector público y privado; sin embargo, aún no cuentan con un marco legal que las regule de forma específica, lo que provoca que en ocasiones se produzcan vulneraciones de los derechos humanos que podrían evitarse con una adecuada regulación. Este artículo avanza en la necesidad de dotar a México de dicha regulación como medio de garantía tanto de los derechos humanos como de los derechos de los médicos y de otros operadores de salud que participan en estas técnicas.
\end{abstract}

PALABRAS CLAVE: Reproducción humana asistida. México. Derechos humanos. Consentimiento bajo información.

\begin{abstract}
Infertility represents a problem in the context of reproductive health; to treat it were developed the techniques of assisted human reproduction, despite medical advances, the practice of techniques is subject to medical, legal and ethical dysfunctions. In Mexico, since the late 1980s assisted reproduction clinics were set up in public and private sector hospitals; however, they do not yet have a legal framework to regulate them in a specific way, which causes human rights violations that could be avoided with adequate regulation. This article advances the need to provide Mexico with such regulation as a means of guaranteeing both human rights and the rights of physicians and other health workers involved in these techniques.
\end{abstract}

KEY WORDS: Human reproductive techniques. México. Human rights. Informed consent.

Correspondencia:

Rosa Verónica Esparza-Pérez

Chabacano 30-5, Barrio San Francisco

Del. Magdalena Contreras

Fecha de recepción: 30-01-2017

C.P. 10500 , Ciudad de México, México

Fecha de aceptación: 28-04-2017

E-mail: esparzaveronica@hotmail.com

DOI:10.24875/GMM.17003159

Gac Med Mex. 2017;153:626-632

Contents available at PubMed

www.gacetamedicademexico.com 


\section{Introducción}

La Organización Mundial de la Salud (OMS) define la infertilidad como el resultado de 2 años de exposición al riesgo de embarazo sin concebir. David Adamson, miembro de la OMS, propuso ante el Comité Internacional de Seguimiento de las Tecnologías de Reproducción Asistida modificar la definición para infertilidad. En palabras de Adamson: «La definición de infertilidad incluye los derechos de todos los individuos para tener una familia, a saber, hombres y mujeres solteras, hombres y mujeres gay. Traza una línea para dejar claro que un individuo tiene derecho a reproducirse tenga o no un compañero sexual»".

La infertilidad representa un problema en el contexto de la salud reproductiva. Las tasas de infertilidad son variables y estudios epidemiológicos refieren que fluctúan entre el 14 y el $16 \%{ }^{2}$. De ahí que en el último cuarto del siglo XX se desarrollaran las técnicas de reproducción humana asistida (TRHA) encaminadas a tratar la infertilidad, y a prevenir y atender enfermedades de origen genético o hereditario. A pesar de los avances médicos, la práctica de las TRHA está sujeta a contingencias médicas, legales y éticas.

\section{Marco normativo de las técnicas de reproducción humana asistida en México}

En 1949, un grupo de ginecólogos, andrólogos, endocrinólogos e investigadores fundaron la Asociación Mexicana para el Estudio de la Esterilidad, con objeto de estudiar los problemas y divulgar los conocimientos sobre la esterilidad y la infertilidad. La asociación para alcanzar su objetivo propuso, entre otras medidas, la creación de clínicas especializadas de esterilidad. Algunos relevantes profesionales de la medicina formaron parte de sus mesas directivas; tal fue el caso de Alfonso Álvarez Bravo, Luis Castelazo Ayala, Francisco Durazo Quiroz, Juan Ramón Argüelles, Javier Soberón Acevedo y Javier Santos González, por mencionar algunos ${ }^{3}$. Es a finales de la década de 1980 que se crean clínicas de reproducción asistida en hospitales públicos y privados ${ }^{4}$; sin embargo, no existe en la actualidad un marco legal que las regule de forma específica.

La primera iniciativa en la materia se presentó en el seno del Poder Legislativo en 1999; aunque a la fecha ningún proyecto ha sido aprobado. Esto da una idea del grado de complejidad que presenta su regulación. En noviembre de 2016, la Cámara de
Senadores aprobó dos minutas, una que regula las TRHA y otra la gestación por sustitución, pero falta que sean votadas por la Cámara de Diputados. Sin embargo, consideramos necesario que sean revisadas, pues ambas contienen disposiciones que, de ser aprobadas, podrían generar cierta vulneración de los derechos de las personas ${ }^{5}$. Habrá que esperar a la aprobación definitiva de las mismas para hacer un análisis exhaustivo de dicha legislación.

Como se ha señalado en otras legislaciones comparadas, la complejidad y la especialización de las TRHA solo deberían abordarse por profesionales capacitados, en establecimientos que requieren infraestructura, equipo, instrumental e insumos específicos. Solo así se puede garantizar la salud de las personas y también el ejercicio responsable de los profesionales de la sanidad que participan en estos tratamientos. En México, corresponde a la Comisión Federal para la Protección contra Riesgos Sanitarios (Cofepris) regular el funcionamiento de los establecimientos públicos y privados donde se practican estos procedimientos; según datos de la Cofepris, hay a la fecha 89 centros autorizados para realizar TRHA ${ }^{6}$.

Los establecimientos públicos que tiene licencia sanitaria para realizar TRHA son el Centro Médico Nacional (CMN) 20 de Noviembre, del Instituto de Seguridad y Servicios Sociales de los Trabajadores (ISSSTE); el Hospital Materno Perinatal Mónica Pretelini, que pertenece al Instituto de Salud del Estado de México; y el Instituto Mexicano de Infertilidad, en Jalisco. El Instituto Nacional de Perinatología Isidro Espinoza de los Reyes (INper) también cuenta con una unidad de reproducción asistida con licencia sanitaria, emitida por la Subsecretaría de Regulación y Fomento Sanitario de la Dirección General de Regulación de los Servicios de Salud, para funcionar como Hospital de Especialidades de Tercer Nivel del Sector Público y contar con la disposición de células germinales para la reproducción asistida ${ }^{7}$.

\section{Programas de reproducción humana asistida en instituciones públicas}

En la mayoría de las instituciones de salud pública de México se atienden problemas de infertilidad a través de procedimientos de baja complejidad -inducción de ovulación e hiperestimulación ovárica con coito programado-. Por referir algunas cifras, en el Instituto Mexicano del Seguro Social mensualmente se practican entre 45 y 60 procedimientos de baja complejidad $^{8}$; y en el Hospital Central Sur de Alta 
Tabla 1. Criterios de admisión a los programas de reproducción asistida en el Inper y el CMN 20 de Noviembre

\begin{tabular}{ll}
\hline INper & CMN 20 de Noviembre \\
Edad & Edad \\
- Mujeres: 35 años & - Mujeres: 35 años \\
- Hombres: 55 años & - Hombres: 55 años \\
Estado civil & Estado civil \\
- Acta de matrimonio o constancia de situación civil & - Parejas constituidas legalmente (presentar acta de matrimonio o \\
Salud & concubinato) \\
- Vida sexual durante 1 año o más sin anticoncepción y sin & Salud \\
haber logrado embarazo & - Pacientes sin anomalías genéticas heredables por sus hijos \\
- Contar con estudio previo de esterilidad y presentar & - Que la pareja no presente alguna enfermedad concomitante, \\
estudios que apoyen el diagnóstico de esterilidad & realizarán una consulta preconcepcional para evaluar el riesgo \\
- Los casos en que la pareja presente azoospermia & potencial de embarazo \\
(falta de espermatozoides) no podrán ser atendidos & - Pacientes con índice de masa corporal < 30; en caso de ser mayor \\
en el Instituto & se le enviará al programa de obesidad e infertilidad, y una vez que \\
Hijos previos & reduzca peso será ingresada al programa de infertilidad en la pareja \\
- No dice nada & Hijos previos \\
& - Con un hijo o ninguno \\
& En caso de cumplir con todos estos requisitos, se evalúan los factores \\
& causantes de infertilidad, y si se contraindica la maternidad se enviará \\
\hline
\end{tabular}

Especialidad de Pemex 9 , de 2006 a 2015 se realizaron 467 tratamientos de baja complejidad. Aun cuando el Hospital de Pemex no cuenta con un programa de reproducción asistida, de existir candidatas a técnicas de alta complejidad se valorarían los casos para su envío a un prestador de servicios contratado, a través de un proceso de licitación pública (de 2006 a 2013 fueron enviadas a servicios contratados 487 pacientes).

Actualmente, las dependencias públicas que realizan anualmente el mayor número de procedimientos de reproducción asistida son el INper y el CMN 20 de Noviembre. El INper, de 2006 a 2015, realizó 2120 procedimientos de baja complejidad y 2308 de alta complejidad $^{10}$. En el CMN 20 de Noviembre se efectuaron, de 2006 a 2015, 523 técnicas de baja complejidad y 1043 de alta complejidad ${ }^{11}$.

\section{Criterios de admisión a los programas de reproducción humana asistida en el INper ${ }^{12}$ y el CMN 20 de Noviembre ${ }^{13}$}

Sin duda, el acceso a la reproducción humana asistida involucra el ejercicio de diversos derechos humanos, entre ellos el de la protección a la salud, a la autonomía reproductiva, a la igualdad, a la no discriminación, a fundar una familia y a beneficiarse de los avances científicos, todos derechos reconocidos en la Constitución Política de los Estados Unidos Mexicanos y en tratados internacionales que México ha ratificado.

En el ámbito internacional, la Corte Interamericana de Derechos Humanos emitió una sentencia del caso
Artavia Murillo y otros vs. Costa Rica ${ }^{14}$. En este proceso, la Corte consideró que la prohibición de practicar la técnica de fertilización in vitro (FIV) en Costa Rica constituyó una injerencia arbitraria en los derechos a la vida privada y familiar, a formar una familia y a la igualdad de las personas con problemas de infertilidad, en tanto el Estado les impidió el acceso a un tratamiento que les hubiera permitido superar su situación de desventaja respecto de la posibilidad de tener descendencia biológica. La Corte resolvió que Costa Rica debía adoptar medidas para dejar sin efecto esa prohibición e incluirla en sus programas de infertilidad, de conformidad con el deber de garantía respecto al principio de no discriminación.

En la tabla 1 se exponen de forma comparativa los criterios de admisión a los programas de reproducción humana asistida que se establecen en el INper y el CMN 20 de Noviembre, para enseguida hacer observaciones sobre ellos.

Tanto el INPer como el CMN 20 de Noviembre establecen como criterio de admisión tener determinada edad, lo que podría interpretarse en determinados casos como una vulneración del principio de igualdad y no discriminación previsto en el artículo $1^{\circ}$ de la Constitución Política de los Estados Unidos Mexicanos, que prohíbe la discriminación por razón de edad. En este sentido podemos referir que, recientemente, un juez de distrito declaró inconstitucional el criterio del ISSSTE que restringe la técnica de reproducción asistida a mujeres mayores de 35 años, y consideró transgredidos los derechos a la vida privada, a fundar una familia y a la salud, en lo relativo al acceso a la tecnología para ejercer tales derechos ${ }^{15}$. El juez que 
resolvió el asunto consideró que, si bien el ISSSTE no puede reparar la ausencia de regulación federal en materia de salud reproductiva, el criterio de no dar este tipo de servicios a mujeres mayores de 35 años viola derechos humanos.

Sobre el mismo criterio, en 2014, la Comisión Nacional de los Derechos Humanos ${ }^{16}$ admitió una queja por la negativa a realizar un procedimiento de reproducción asistida a una paciente que rebasaba la edad de 35 años. La autoridad señalada como responsable fue el ISSSTE y el asunto fue concluido como solucionado durante el proceso de su tramitación, toda vez que la autoridad autorizó que se hiciera el tratamiento que requería la quejosa.

Por otra parte, respecto al criterio de que las personas, para ser admitidas, deban estar unidas en matrimonio o ser una pareja constituida legalmente, viola el principio de no discriminación motivada por el estado civil de las personas, en términos de lo que establece el artículo $1^{\circ}$ de nuestra Carta Magna y diversos tratados internacionales de los que el Estado mexicano es parte, en la medida en la que este criterio de admisión deja fuera a personas que desean concebir en solitario, y a parejas del mismo sexo, mediante las técnicas de reproducción asistida. Podría pensarse que las parejas del mismo sexo que acreditaran su estado civil con acta de matrimonio ${ }^{17}$ deberían ser admitidas en sus programas de reproducción asistida, pero ambas instituciones requieren además que la pareja sea infértil, criterio que no podría cumplir una pareja homosexual.

Mary Warnock señala, sobre el caso de homosexuales que piden asistencia para la reproducción, que si bien no hay ley positiva que otorgue a la gente el derecho a tener hijos, tampoco hay una ley que prohíba a los homosexuales tenerlos, y agrega que si son automáticamente rechazados de las clínicas de fertilidad, aun así procederán por su cuenta. $Y$ este es un camino que hará inevitable que esta práctica quede envuelta en el secreto y a su vez garantizará que los juicios sobre su moralidad o anormalidad continúen basándose en la ignorancia ${ }^{18}$.

En México, el 30 de enero de 2017 entró en vigor la jurisprudencia de la Suprema Corte de Justicia de la Nación sobre la vida familiar entre parejas del mismo sexo, que considera el derecho que tienen las personas del mismo sexo a utilizar los medios derivados de los avances científicos para tener hijos. El criterio de la Corte, basado en las consideraciones del Tribunal Europeo de Derechos Humanos sobre la similitud entre las parejas homosexuales y heterosexuales en cuanto a su capacidad para desarrollar una vida familiar, se plasma en el siguiente párrafo: «La Primera Sala entiende que la vida familiar entre personas del mismo sexo no se limita únicamente a la vida en pareja, sino que puede extenderse a la procreación y a la crianza de niños y niñas según la decisión de los padres. Así existen parejas del mismo sexo que hacen vida familliar con niños y niñas procreados o adoptados por alguno de ellos, o parejas que utilizan los medios derivados de los avances científicos para procrear ${ }^{19}$.

A pesar de que el criterio de la Corte no se refiere a las personas solteras, sino que alude a parejas, sin duda será importante para promover el acceso igualitario a procedimientos de reproducción asistida en México.

Otro caso en el que una pareja no estéril podría solicitar un tratamiento de reproducción asistida y que, atendiendo a los criterios que actualmente utilizan tanto el INper como el CMN 20 de Noviembre no podría ser admitida, es el que refiere la misma autora Mary Warnock ${ }^{20}$, cuando cualquier niño que vayan a tener juntos cuente con un alto porcentaje de riesgo de padecer una seria enfermedad hereditaria. Podrían seleccionarse los embriones que no estén afectados por la enfermedad para ser transferidos en el útero de la mujer. En este caso, la pareja no podría ingresar al programa de infertilidad al no contar con un diagnóstico de esterilidad, pero tampoco cumpliría con el criterio de ser «pacientes sin anomalías genéticas heredables por sus hijos" que se establece en el CMN 20 de Noviembre.

Con relación a limitar el ingreso a los pacientes sin anomalías genéticas heredables por sus hijos y que la pareja no presente alguna enfermedad concomitante -aspecto que establece el CMN 20 de Noviembre-, si bien es importante considerar que la naturaleza jurídica de los derechos reproductivos tiene que conformarse mediante un equilibrio entre la libertad y la responsabilidad, sin duda el límite claro para los mismos son los derechos y las necesidades de los hijos nacidos y futuros, así como el bien común ${ }^{21}$. No obstante, este criterio resulta ambiguo y podría dejar a los pacientes en indefensión frente a una posible decisión arbitraria por parte de las autoridades. Por ejemplo, ¿qué tipo de enfermedades genéticas y concomitantes?, ¿enfermedades heteditarias graves de posible aparición tardía o de aparición precoz, multifactoriales o de expresión fenotípica variable? Un ejemplo sería la neurofibromatosis, enfermedad de expresión fenotípica variable caracterizada por 
anomalías del desarrollo de músculos, huesos y vísceras. No es dominante el padecimiento de la enfermedad por la descendencia, y en el supuesto de desarrollar su sintomatología podrá ser grave o no, dependiendo también de factores multifactoriales que influirán a lo largo de su vida ${ }^{22}$. Y si se tratara de una enferemedad genética no grave y susceptible de tratamiento curativo posnatal, ¿también estaría justificado negar el acceso? Si fuera alguna enfermedad hereditaria ligada al sexo del bebé, ¿se ofrecería a las parejas realizar diagnóstico genético preimplantatorio para la detección de alteraciones específicas, genéticas, estructurales o cromosómicas, y seleccionar el sexo para evitar la transmisión de la enfermedad a la descendencia o bien recurrir a la donación de gametos o embriones? Por ejemplo, la retinosis pigmentaria, enfermedad que podría transmitirse a la descendencia con una probabilidad del $100 \%$ en el caso de que sus hijos fueran del sexo masculino ${ }^{23}$.

Desde el punto de vista médico, ¿en qué casos se podría hablar realmente de la existencia de una enfermedad grave que justifique negar el acceso al servicio de reproducción asistida? ¿Y qué debe entenderse por enfermedad grave? ¿Quién decide cuáles son esas enfermedades y bajo qué criterios? Estos aspectos son importantes para una justificación moral y jurídica que posibilite negar el acceso a cualquier TRHA o al diagnóstico genético preimplantatorio ${ }^{24}$.

Por último, en el CMN 20 de Noviembre se limita el acceso a pacientes con un hijo o ninguno, situación que podría colisionar con los derechos a la vida privada y a fundar una familia. El artículo $4^{\circ}$ de nuestra Carta Magna establece que toda persona tiene derecho a decidir de manera libre, responsable e informada sobre el número y el esparcimiento de sus hijos. El derecho a la autonomía reproductiva está reconocido también en el artículo 16 (e) de la Convención para la Eliminación de todas las Formas de Discriminación contra la Mujer, según el cual las mujeres gozan del derecho «a decidir libre y responsablemente el número de sus hijos y el intervalo entre los nacimientos, y a tener acceso a la información, la educación y los medios que les permitan ejercer estos derechos".

A modo de conclusión de este apartado podemos decir que ante la falta de una normatividad específica que regule los aspectos de la reproducción humana asistida en México se promueve que las instituciones de salud pública y privada operen conforme a criterios internos, situación que provoca inseguridad jurídica tanto del personal médico como de los pacientes.
Ingrid Brena ${ }^{25}$ señala al respecto que «resulta necesario insistir en que no solo las legislaciones, sino también cualquier toma de decisiones -tanto administrativas como judiciales-, deben fundamentarse en información científica que permita la construcción de una convivencia respetuosa dirigida hacia un objetivo primordial: el respeto a los derechos humanos involucrados con los derechos reproductivos».

\section{Consentimiento bajo información}

Cano Valle ${ }^{26}$ define el consentimiento bajo infomación (CBI) como «un proceso mediante el cual se garantiza por escrito, después de haber recibido y comprendido toda la información necesaria y pertinente, el paciente ha expresado voluntariamente su intención de participar en cualquier investigación, o su autorización para que sobre él se efectúen procedimientos diagnósticos, tratamientos médicos o intervenciones quirúrgicas de cualquier tipo, que suponen molestias, riesgos o inconvenientes que previsiblemente pueden afectar su salud o su dignidad, así como las alternativas posibles, derechos, obligaciones y responsabiidades".

La carta del CBI, de acuerdo con la Norma Oficial Mexicana NOM-004-SSA3-2012 (del expediente clíni$\mathrm{co})^{27}$, se define como el documento escrito, signado por el paciente o su representante legal o familiar más cercano en vínculo, mediante el cual se acepta un procedimiento médico o quirúrgico con fines diagnósticos, terapéuticos, rehabilitatorios, paliativos o de investigación, una vez que se ha recibido información de los riesgos y beneficios esperados para el paciente.

\section{Consentimiento bajo información en el INper y el CMN 20 de Noviembre}

En este apartado referiremos algunos aspectos de los escritos de CBI sobre FIV y transferencia embrionaria (TE) que se utilizan en el INper y el CMN 20 de Noviembre. Con relación a la obtención del CBI para pacientes foráneas del CMN 20 de Noviembre, llama la atención que el formato de consentimiento se les hace llegar a los usuarios vía fax, quienes lo deberán entregar firmado en el momento que sean citados para la apertura del expediente ${ }^{28}$. Al respecto, la NOM-004-SSA3-2012 (del expediente clínico) establece que, previo a la firma del documento, el paciente deberá recibir información sobre los riesgos y beneficios del procedimiento que se va a realizar. En 
este caso, no se advierte que el médico tratante brinde, a los pacientes foráneos, orientación suficiente con antelación a la firma del documento de consentimiento.

Para obtener el CBI será necesario que la información se proporcione en forma oral y personal; solo así se pueden conocer las necesidades de los usuarios y en consecuencia brindarles, de manera comprensible, asesoramiento suficiente sobre los distintos aspectos e implicaciones posibles, así como sobre los resultados y los riesgos que los procedimientos conllevan. En todos los casos, la obtención del consentimiento deberá ser un proceso continuo entre el personal de salud y el usuario que, en algunos supuestos, deberá consolidarse en un documento ${ }^{29}$.

Otro aspecto importante es la información que se proporciona a las pacientes con relación al número de embriones a transferir en cada ciclo de reproducción asistida. Sobre esto es fundamental que el equipo médico comunique a la paciente acerca del número máximo de embriones que se le pueden transferir por ciclo, para mejorar las posibilidades de embarazo, sin poner en riesgo su salud ni incrementar las posibilidades de un embarazo múltiple. Sin embargo, en el formato de $\mathrm{CBI}$ que utiliza el INper no se advierte a la usuaria sobre el número de embriones que se le transferirán, aspecto que debería consultársele a fin de garantizar su derecho a decidir. Asimismo, en el CMN 20 de Noviembre, en el formato de CBI se especifica que el número de embriones a transferir será decisión del equipo médico a cargo, siempre buscando un equilibrio entre probabilidad de embarazo y minimizar los riesgos de embarazo múltiple.

Sin duda, es necesario establecer un límite de embriones a transferir; sin embargo, la decisión final, sin rebasar el máximo de embriones sugerido por el personal médico, compete a la paciente. Lo anterior, debido a que los valores u objetivos de las personas varían, y la mejor elección no siempre es la que prioriza a la salud, sino la que prioriza el máximo bienestar de acuerdo con los valores u objetivos de cada persona. Por lo tanto, no es ya el médico el único que decide la mejor alternativa ${ }^{30}$; la labor del equipo médico debiera ser la de proporcionar la información que requieran los pacientes, y en el caso de existir un dilema bioético, consultar a su comité interdisciplinario para conocer las alternativas o sugerencias dirigidas a la toma de decisiones.

Ahora bien, con relación al consentimiento para el destino de embriones supernumerarios de TRHA, el asunto es complejo, pese a que teóricamente estos embriones pudieran tener tres destinos: la criopreservación (para uso propio), el descarte o la donación (con fines asistenciales o clínicos, fines de investigación o fines docentes) ${ }^{31}$. En México, al no haber normatividad que regule sobre la utilización de embriones supernumerarios, tanto en el INper como en el CMN 20 de Noviembre, el único destino final que en este momento se puede dar a los embriones criopreservados supernumerarios es para fines reproductivos de uso exclusivo de la pareja ${ }^{32}$. Pero en el caso de que decidan no disponer de ellos para su propia reproducción, dichos embriones quedarían congelados por tiempo indeterminado ${ }^{33}$. Así, desde 2006 hasta la fecha, el laboratorio de reproducción asistida del CMN 20 de Noviembre tiene bajo su resguardo 397 embriones supernumerarios criopreservados; por su parte, el INper, de 2010 a 2016, mantiene en criopreservación 657 embriones ${ }^{34,35}$.

Por último, respecto a la información que se proporciona a los pacientes en el formato de CBI sobre la eficiencia del procedimiento de FIV/TE, tanto el INper como el CMN 20 de Noviembre utilizan como referencia resultados obtenidos en Latinoamérica y reportados en el Registro Latinoamericano de Reproducción Asistida ${ }^{36,37}$.

Sobre la eficiencia de TRHA, en el mismo formato de consentimiento se informa a los pacientes de que esta se mide por la proporción (tasa) de mujeres que logran un embarazo luego de haber transferido embriones al útero. Considerando que la tasa (proporción) de abortos espontáneos es de aproximadamente un $15-25 \%$, la medida más real de evaluar la eficiencia es midiendo la tasa de partos o de nacidos vivos $^{38,39}$. En este momento, el INper y el CMN 20 de Noviembre no cuentan con estimaciones realistas de éxito en sus programas de reproducción asistida, debido a que un alto porcentaje de las pacientes que logran el embarazo con TRHA no continúan el seguimiento obstétrico en esas instituciones. Al efecto de subsanar este aspecto, el INper pretende implementar el uso del expediente clínico electrónico para realizar el seguimiento de las pacientes ${ }^{40}$. Por su parte, el Servicio de Reproducción Humana, en colaboración con el Servicio de Planeación del CMN 20 de Noviembre, trabaja en la elaboración de indicadores para medir la eficiencia y la calidad del servicio ${ }^{41}$.

\section{Conclusión}

De lo referido con anterioridad se advierte la urgente necesidad de que los órganos legislativos en 
México desarrollen una regulación integral en materia de reproducción humana asistida que sea respetuosa de los derechos humanos, garantice y proteja la actividad de los profesionales de la salud, y propicie el avance de la ciencia.

Es preciso establecer directrices para que los establecimientos de salud, tanto públicos como privados, dirijan su actuación con apego a derecho y se garantice un acceso justo, equitativo y con un trato digno y de calidad.

El destino de los recursos sanitarios y la definición de los criterios para la solución de prioridades en caso de que una demanda excesiva impida que se vean satisfechas todas las solicitudes de tratamiento, constituye sin duda un problema para la salud pública. Sin embargo, ello no exime a las instituciones para que la atención y el servicio que presten sean compatibles con los derechos humanos, y que las mismas implementen mecanismos que permitan una distribución equitativa de estos servicios.

Por otra parte, es necesario establecer los requisitos del $\mathrm{CBI}$ en materia de reproducción asistida para proteger el derecho de los usuarios, asegurar que tomen decisiones de manera autónoma, libre e informada, y su obtención no constituya un mero requisito formal.

Por último, es fundamental la intervención de los Comités Hospitalarios de Bioética y de los Comités de Ética para lograr el consenso en la toma de decisiones, basado en el reconocimiento de la dignidad de los individuos, en la autonomía de los pacientes y en la protección del interés superior del menor.

\section{Bibliografía}

1. Bodkin $\mathrm{H}$. Single man will get the right to start a family under new definition of infertility. The Thelegraph, 20 de octubre de 2016. (Consultado el 29 de octubre de 2016.) Disponible en: http://www.telegraph.co.uk/ news/2016/10/19/single-men-will-get-the-right-to-start-a-family-undernew-defini

2. Diagnóstico de la pareja infértil y tratamiento con técnicas de baja complejidad. México: Instituto Mexicano del Seguro Social; 2013. p. 8.

3. Septién González JM. Historia de la ginecología y la obstetricia en México. México: Editorial Ciencia y Cultura de México; 1986. p. 662-5.

4. Cardaci D, Sánchez Bringas A. "Hasta que lo alcancemos..." Producción académica sobre la reproducción asistida en publicaciones mexicanas. Alteridades. 2009;19:24

5. Palacios $\mathrm{C}$. Una ley que prohíbe embarazos y trasplantes. Animal Político [México], 14 de diciembre de 2016. (Consultado el 16 de diciembre de 2016.) Disponible en: animalpolitico.com/blogueros-una-vida-exami- nada-reflexiones-bioeticas/2016/12/14/una-propuesta-ley-prohibe-embarazos-trasplantes

6. Sistema de Acceso a la Información Pública: Infomex, folio 1215100132716.

7. Ibidem, folio 1225000012416 .

8. Ibidem, folio 0064102296416 .

9. Ibidem, folio 1857200288816 .

10. Ibidem, folio 1225000004816 .

11. Ibidem, folio 0063700517416 .

12. Detección y tratamiento integral de la pareja con problemas de infertilidad. Requisitos para obtener el servicio, criterios de aceptación y documentación requerida por trabajo social. Información publicada en el sitio web del INper. (Consultado el 4 de octubre de 2016.) Disponible en: http://www.inper.mx/TramitesRequisitos/ProblemasInfertilidad.html

13. Sistema de Acceso a la Información Pública: Infomex, folio 0063700116616.

14. Corte Interamericana de Derechos Humanos. Caso Artavia Murillo y otros ("Fertilización in vitro") vs Costa Rica, Excepciones Preliminares, Fondo y Reparaciones y Costas, 28 de noviembre de 2012. Disponible en: http://www.corteidh.or.cr/docs/casos/articulos/seriec_257_esp.pdf

15. Langer A. Limitar la reproducción asistida va contra los derechos humanos. El Economista [México], 17 de agosto de 2016. (Consultado el 16 de noviembre de 2016.) Disponible en: http://eleconomista.com.mx/entretenimiento/2016/08/17/limitar-reproduccion-asistida-va-contra-derechos-humanos

16. Sistema de Acceso a la Información Pública: Infomex, folio: 3510000009316.

17. Recomendación General No. 23/2015 sobre el Matrimonio Igualitario. (Consultado el 15 de octubre de 2016.) Disponible en: http://www.cndh. org.mx/sites/all/doc/Recomendaciones/generales/RecGral_023.pdf

18. Warnock M. Fabricando bebés. ¿Existe un derecho a tener hijos? España: Gedisa; 2004, p. 71, 84.

19. Tesis: 1 a J. $8 / 2017\left(10^{a}\right)$, Semanario Judicial de la Federación, Décima Época, enero de 2017, registro 201353.

20. Warnock M. Op. cit. p. 68

21. Abellán F. Selección genética de embriones. España: Comares; 2007. p. 147.

22. Ibidem, p. 114-120.

23. Ibidem, p. 125.

24. Ibidem, p. 186.

25. Brena Sesma I. La sentencia de la Corte Interamericana de Derechos Humanos, Caso Artavia Murillo y otros (fecundación in vitro) contra Costa Rica. Un avance en el pensamiento laico en Latinoamérica. En: Carbonell Sánchez M, Fix-Fierro HF, Valadés D, coordinadores. Estado constitucional, derechos humanos, justica y vida universitaria. Estudio homenaje a Jorge Carpizo. Derechos Humanos. Tomo V, vol. 1. México: UNAM; 2015. p. 196-7.

26. Cano Valle F. Bioética. Temas humanísticos y jurídicos. México: UNAM; 2005. p. 25.

27. Norma Oficial Mexicana NOM-004-SSA3-2012, del expediente clínico. Diario Oficial de la Federación del 15 de octubre de 2012.

28. Sistema de Acceso a la Información Pública: Infomex, folio: 0063700116616.

29. Guía Nacional para la Integración y el Funcionamiento de los Comités Hospitalarios de Bioética. Secretaría de Salud; 2015. Disponible en: h?p://www.conbioe<ca-mexico.salud.gob.mx/descargas/pdf/registrocomites/Guia_CHB_Final_Paginada_con_forros.pdf

30. Ibidem, p. 48.

31. Álvarez-Díaz JA. Donación de embriones en países desarrollados. Gac Med Mex. 2010;146:229.

32. Sistema de Acceso a la Información Pública: Infomex, folio: 1225000000617.

33. Ibidem, folio: 0063700028817 .

34. Idem.

35. Ibidem, folio: 1225000000617.

36. Ibidem, folio: 1225000004816 .

37. Ibidem, folio: 0063700116616 .

38. Idem.

39. Ibidem, folio: 1225000004816 .

40. Idem.

41. Ibidem, folio: 0063700517416 . 